\title{
BMR
}

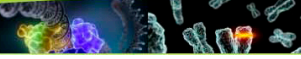

\section{Role of MTHFR C677T and MTR A2756G polymorphisms in thyroid and breast cancer development}

T. Zara-Lopes ${ }^{1}$ A.P A Gimenez-Martins ${ }^{1}$, C.H.V. Nascimento-Filho ${ }^{1}$ M.M.U. Castanhole-Nunes ${ }^{1}$, A.L.S. Galbiatti-Dias ${ }^{1}$, J.A. Padovani-Júnior ${ }^{2}$, J.V. Maniglia ${ }^{2}$, J.L.E. Francisco ${ }^{3}$, E.C. Pavarino ${ }^{1,4}$ and E.M. Goloni-Bertollo ${ }^{1,4}$

${ }^{1}$ Unidade de Pesquisa em Genética e Biologia Molecular, Faculdade de Medicina de São José do Rio Preto, São José do Rio Preto, SP, Brasil

${ }^{2}$ Departamento de Otorrinolaringologia e Cirurgia de Cabeça e Pescoço, Hospital de Base de São José do Rio Preto, São José do Rio Preto, SP, Brasil ${ }^{3}$ Departamento de Ginecologia e Obstetrícia, Hospital de Base de São José do Rio Preto, São José do Rio Preto, SP, Brasil ${ }^{4}$ Departamento de Biologia Molecular, Faculdade de Medicina de São José do Rio Preto, São José do Rio Preto, SP, Brasil

Corresponding author: E.M. Goloni-Bertollo

E-mail: itairine_@hotmail.com / eny.goloni@famerp.br

Genet. Mol. Res. 15 (2): gmr.15028222

Received December 7, 2015

Accepted January 18, 2016

Published May 6, 2016

DOI http://dx.doi.org/10.4238/gmr.15028222

ABSTRACT. Folate metabolism is essential for DNA synthesis and repair. Alterations in genes that participate in folate metabolism can be associated with several types of malignant neoplasms, including thyroid and breast cancer. In the present case-control study, we examined the association between methylenetetrahydrofolate reductase (MTHFR C677T, rs1801133) and methionine synthase (MTR A2756G, rs1805087) polymorphisms and risk for thyroid and breast cancer. Polymerase chain reaction-restriction fragment length technique was used to determine 
the specific genotypes in the genes of interest. Statistical analysis was performed by multiple logistic regression test. We found an association between MTHFR C677T polymorphism and risks to both thyroid (OR $=2.50 ; 95 \% \mathrm{CI}=1.15-5.46 ; \mathrm{P}=0.02)$ and breast cancer $(\mathrm{OR}=2.53$; $95 \% \mathrm{CI}=1.08-5.93 ; \mathrm{P}=0.03$ ). Tobacco consumption and high body mass index were also associated with thyroid cancer. In addition, increased age ( $\geq 50$ years) and alcohol consumption were found to be associated with breast cancer. Our results indicated that MTHFR C677T is significantly associated with thyroid and breast cancer risks. Thus, these factors may be used as potential prognostic markers for thyroid and breast cancers.

Key words: Breast cancer; Folate; Genes; Genetic polymorphism; Thyroid cancer

\section{INTRODUCTION}

Thyroid and breast cancers predominantly affect women. Thyroid cancer is the most common malignancy of the endocrine system. It is estimated that there are approximately 300,000 new cases of thyroid cancer worldwide. Of these, 230,000 are females. The estimate for Breast cancer is approximately 57,120 new cases, 56.09 cases per 100,000 women, representing $25 \%$ of total cancers diagnosed in women (INCA, 2014). The number of cases for these cancers has been steadily increasing. Breast Cancer ranks as the second most common cause of death by cancer in women (INCA 2014). Multiple risk factors such as hormones, family history of cancer, tobacco and alcohol consumption, obesity, poor diet in folic acid, and genetic variations all contribute to the development of thyroid and breast cancer (Gong et al., 2015). Studies examining single nucleotide polymorphisms (SNPs) in folate metabolism have been performed in several cancer types, however, the currently available literature is inconsistent and contradictory, suggesting that further studies are required in this field of research (Zhong et al., 2013; Yang et al., 2014). In thyroid cancer research, the folate pathways are poorly studied (Fard-Esfahani et al., 2011; Ozdemir et al., 2012). Low folate levels cause genomic instability through DNA synthesis, methylation, and alterations to repair mechanisms. Consequently, low folate levels can induce carcinogenesis (Alshatwi, 2010; Yang et al., 2013; Taflin et al., 2014). Several enzymes, including methylenetetrahydrofolate reductase (MTHFR) and methionine synthase (MTR) regulate this metabolism (Yin et al., 2004; Taflin et al., 2014). The MTHFR enzyme, encoded by the MTHFR gene, is responsible for catalyzing the irreversible reaction of 5,10-methylenetetrahydrofolate to 5-methyltetrahydrofolate, which is involved in DNA methylation, an important factor gene expression regulation. Alterations in DNA methylation due to polymorphisms in the MTHFR gene may be associated with cancer development (Yin et al., 2004; Alshatwi, 2010; Jiang-hua et al., 2014). The MTR enzyme is encoded by the MTR gene, and is responsible for catalyzing homocysteine remethylation to form methionine using the cofactor vitamin $\mathrm{B}_{12}$. Polymorphisms in this gene increase homocysteine in the plasma, resulting in changes to the folate pathway, and can induce carcinogenesis (Weiner et al., 2012; Jiang-hua et al., 2014). According to several studies, MTHFR C677T and MTR A2756G polymorphisms are able to change folate metabolism, which is important for DNA synthesis and methylation, as well as genomic stability (Yin et al., 2004; Alshatwi, 2010; Weiner et al., 2012; Jiang-hua et al., 2014). The aim of the present study was to investigate the associations between MTHFR C677T and MTR A2756G polymorphisms involved in folate metabolism 
and thyroid and breast cancers. We also wanted to determine the interaction between these polymorphisms and other risk factors (age, alcohol consumption, tobacco, and body mass index $[\mathrm{BMI}])$ in the disease.

\section{MATERIAL AND METHODS}

\section{Subjects}

A total of 344 women were evaluated in this case-control study; 200 patients (100 thyroid cancer and 100 breast cancer) and 144 healthy women without history of cancer were recruited between January 2013 and January 2015.

Patients with thyroid and breast cancer were admitted to Hospital de Base. The hospital is located in the city of São José do Rio Preto, São Paulo State, Brazil. The physicians responsible made the definitive diagnoses by examining the results from imaging studies, histopathological analysis, and biopsies. Patients with other neoplasms were excluded from the case group. The control group was comprised of healthy women. Women were excluded from the study if they had a family history of cancer. All individuals signed the written informed consent form prior to participation in the study. This study was approved by the Faculdade de Medicina de São José do Rio Preto (FAMERP) Research Ethics Committee (Thyroid cancer REC approval: 20187413.8.0000.5415; Breast Cancer REC approval: 04069612.1.0000.5415).

Sample calculation was performed according the reports of Ozdemir et al. (2012) and Diakite et al. (2012). This study is the first to evaluate polymorphisms in folate metabolism with regard to thyroid cancer development in the Brazilian population.

\section{Genotyping}

Peripheral blood samples were collected from all the subjects using EDTA (ethylenediamine tetraacetic acid)-containing tubes. Genomic DNA was extracted by methods described by Miller et al. (1988) with modifications. The MTHFR C677T (rs1801133) and MTR A2756G (rs1805087) polymorphisms were determined by PCR-RFLP using the following primers: MTHFR C677T, sense 5'-TGA AGG AGA AGG TGT CTG CGG GA3', anti-sense 5'-AGG ACG GTG CGG TGA GAG TG-3'; MTR A2756G, sense 5'-CCA GGG TGC CAG GTA TAC AG-3', anti-sense 5'-GCC TTT TAC ACT CCT CAA AAC-3'. Genotyping of MTHFR C677T polymorphism was accomplished by the restriction enzyme HinfI. The resulting fragments were: $198 \mathrm{bp}$ (C allele), 175, and $23 \mathrm{bp}$ (T allele). The MTR A2756G polymorphism was genotyped using the restriction enzyme HaeIII. The resulting fragments were 413, 85 bp (A allele), 290, 123, and 85 bp (G allele) (Galbiatti et al., 2010; Rodrigues et al., 2010). Genotyping confirmation was carried out randomly in $10 \%$ of samples from each group, and we observed $100 \%$ concordance.

\section{Statistical analysis}

The Hardy-Weinberg equilibrium was evaluated by chi-square tests using the BioEstat 5.4 software. Multiple regression logistic test was performed by the Minitab/Version 14.0 software, adjusting for age (thyroid cancer-reference $<49$ years and breast cancer-reference $<50$ years), alcohol consumption (reference: no alcohol consumption), tobacco smoking (reference: non-smoking), BMI (reference <24.9), MTHFR C677T genotype (reference: genotype CC-CT), 
and MTR A2756G genotype (reference: genotype AA-AG). In this study, we defined smokers as those who smoked $>100$ cigarettes in their lifetime, and drinkers as those who has at least 4 drinks per week. One drink is equivalent to $30 \mathrm{~mL}$ liquor, $102 \mathrm{~mL}$ wine, and $340 \mathrm{~mL}$ beer (Carpenter et al., 1998; Kjaerheim et al., 1998; Ahrendt et al., 2000). Subjects with BMI $\geq 25.0$ were considered overweight (Naushad et al., 2011; James et al., 2015).

The SNPstats online computer program was used to analyze the polymorphisms' effect in the following models: 1) codominant (heterozygous $v s$ homozygous wild type and polymorphic homozygous $v s$ homozygous wild type), 2) dominant (heterozygous more polymorphic homozygous $v s$ homozygous wild type), 3) recessive (polymorphic homozygous vs homozygous wild type more heterozygous), 4) overdominant (wild homozygous vs heterozygous more polymorphic homozygote), and 5) additive (weight polymorphic homozygote $v$ s heterozygote 2 more homozygous wild-type).

SNPstat online computer program was used to investigate the interaction between MTHFR C677T and MTR A2756G polymorphisms, as well as the relationship between alcohol consumption, tobacco smoking, and BMI and the risk of thyroid and breast cancer. The results of both analyses were presented in odds ratio (OR) with 95\% confidence interval (CI - 95\%), and a $P$ value of $<0.05$ was considered to be significant.

\section{RESULTS}

Tables 1 and 2 show the association between MTHFR C677T and MTR A2756G polymorphisms and the risk of thyroid and breast cancer according to heritage models. The 677TT genotype was associated with increased risk for both thyroid cancer $(\mathrm{OR}=2.50$; $95 \% \mathrm{CI}=1.15-5.46 ; \mathrm{P}=0.02)$ and breast cancer $(\mathrm{OR}=2.53 ; 95 \% \mathrm{CI}=1.08-5.93 ; \mathrm{P}=0.03)$ development. We observed no associations in other models. No statistical significance was observed between MTR A2756G polymorphism and the risk of thyroid and breast cancers.

\begin{tabular}{|c|c|c|c|c|c|c|}
\hline SNP & Model & Genotype & Cases [N (\%)] & Controls [N (\%)] & OR $(95 \% \mathrm{CI})$ & P value \\
\hline \multirow[t]{5}{*}{ MTHFR C677T } & Codominant & $\begin{array}{c}\mathrm{C} / \mathrm{C} \\
\mathrm{C} / \mathrm{T} \\
\mathrm{T} / \mathrm{T} \\
\text { Allele C } \\
\text { Allele T } \\
\text { HWE test }\end{array}$ & $\begin{array}{c}40(40) \\
41(41) \\
19(19) \\
121(60.5) \\
79(39.5) \\
P=0.15 \\
\end{array}$ & $\begin{array}{c}66(45.83) \\
65(45.13) \\
13(9.04) \\
197(68.4) \\
91(31.6) \\
P=0.59\end{array}$ & $\begin{array}{c}1.00 \text { (ref) } \\
1.10(0.62-1.96) \\
2.63(1.14-6.04)\end{array}$ & 0.06 \\
\hline & Dominant & $\begin{array}{c}\mathrm{C} / \mathrm{C} \\
\mathrm{C} / \mathrm{T}-\mathrm{T} / \mathrm{T}\end{array}$ & $\begin{array}{l}40(40) \\
60(60)\end{array}$ & $\begin{array}{l}66(45.83) \\
78(54.17)\end{array}$ & $\begin{array}{c}1.0 \text { (ref) } \\
1.36(0.79-2.33)\end{array}$ & 0.26 \\
\hline & Recessive & $\begin{array}{c}\mathrm{C} / \mathrm{C}-\mathrm{C} / \mathrm{T} \\
\mathrm{T} / \mathrm{T}\end{array}$ & $\begin{array}{l}81(81) \\
19(19) \\
\end{array}$ & $\begin{array}{c}131(90.96) \\
13(9.04)\end{array}$ & $\begin{array}{c}1.0(\mathrm{ref}) \\
2.50(1.15-5.46)\end{array}$ & $0.02^{*}$ \\
\hline & Overdominant & $\begin{array}{c}\mathrm{C} / \mathrm{C}-\mathrm{T} / \mathrm{T} \\
\mathrm{C} / \mathrm{T}\end{array}$ & $\begin{array}{l}59(59) \\
41(41)\end{array}$ & $\begin{array}{l}79(54.9) \\
65(45.1)\end{array}$ & $\begin{array}{c}1.0 \text { (ref) } \\
0.87(0.51-1.49)\end{array}$ & 0.62 \\
\hline & \begin{tabular}{|l} 
Additive \\
\end{tabular} & - & - & - & $1.47(1.00-2.16)$ & 0.05 \\
\hline \multirow[t]{5}{*}{ MTR A2756G } & Codominant & $\begin{array}{c}\mathrm{A} / \mathrm{A} \\
\mathrm{A} / \mathrm{G} \\
\mathrm{G} / \mathrm{G} \\
\text { Allele A } \\
\text { Allele G } \\
\text { HWE test }\end{array}$ & $\begin{array}{c}63(63) \\
28(28) \\
9(9) \\
154(77) \\
46(23) \\
\mathrm{P}=0.03 \\
\end{array}$ & $\begin{array}{c}88(61.11) \\
50(34.72) \\
6(4.17) \\
226(78.4) \\
62(21.6) \\
P=0.73\end{array}$ & $\begin{array}{c}1.00 \text { (ref) } \\
0.82(0.46-1.47) \\
1.82(0.60-5.50)\end{array}$ & 0.39 \\
\hline & Dominant & $\begin{array}{c}\mathrm{A} / \mathrm{A} \\
\mathrm{A} / \mathrm{G}-\mathrm{G} / \mathrm{G}\end{array}$ & $\begin{array}{l}63(63) \\
37(37) \\
\end{array}$ & $\begin{array}{l}88(61.11) \\
56(38.89) \\
\end{array}$ & $\begin{array}{c}1.00 \text { (ref) } \\
0.94(0.55-1.62)\end{array}$ & 0.83 \\
\hline & Recessive & $\begin{array}{c}\mathrm{A} / \mathrm{A}-\mathrm{A} / \mathrm{G} \\
\mathrm{G} / \mathrm{G}\end{array}$ & $\begin{array}{c}91(91) \\
9(9)\end{array}$ & $\begin{array}{c}138(95.83) \\
6(4.17)\end{array}$ & $\begin{array}{c}1.0(\mathrm{ref}) \\
1.93(0.65-5.76)\end{array}$ & 0.23 \\
\hline & Overdominant & $\begin{array}{c}\mathrm{A} / \mathrm{A}-\mathrm{G} / \mathrm{G} \\
\mathrm{A} / \mathrm{G}\end{array}$ & $\begin{array}{l}72(72) \\
28(28)\end{array}$ & $\begin{array}{l}95(66) \\
49(34)\end{array}$ & $\begin{array}{c}1.0(\mathrm{ref}) \\
0.78(0.44-1.38)\end{array}$ & 0.40 \\
\hline & Additive & - & - & - & $1.07(0.70-1.64)$ & 0.76 \\
\hline
\end{tabular}

$\mathrm{OR}=$ odds ratio; adjusted for age, alcohol and smoking consumption, BMI (Body-mass index) and polymorphisms; HWE $=$ Hardy-Weinberg equilibrium; $*$ P value statistically significant. 
Table 2. Association between MTHFR C677T and MTR A2756G polymorphisms and breast cancer.

\begin{tabular}{|c|c|c|c|c|c|c|}
\hline$\overline{\mathrm{SNP}}$ & Model & Genotype & Cases [N (\%)] & Controls [N (\%)] & OR $(95 \% \mathrm{CI})$ & $P$ value \\
\hline \multirow[t]{5}{*}{ MTHFR C677T } & Codominant & $\begin{array}{c}\mathrm{C} / \mathrm{C} \\
\mathrm{C} / \mathrm{T} \\
\mathrm{T} / \mathrm{T} \\
\text { Allele C } \\
\text { Allele T } \\
\text { HWE test }\end{array}$ & $\begin{array}{c}35(35) \\
48(48) \\
17(17) \\
118(59) \\
82(41) \\
\mathrm{P}=0.93\end{array}$ & $\begin{array}{c}66(45.83) \\
65(45.13) \\
13(9.04) \\
197(68.4) \\
91(31.6) \\
\mathrm{P}=0.59\end{array}$ & $\begin{array}{c}1.00 \text { (ref) } \\
1.09(0.59-2.03) \\
2.65(1.07-6.58)\end{array}$ & 0.09 \\
\hline & Dominant & $\begin{array}{c}\mathrm{C} / \mathrm{C} \\
\mathrm{C} / \mathrm{T}-\mathrm{T} / \mathrm{T}\end{array}$ & $\begin{array}{l}35(35) \\
65(65) \\
\end{array}$ & $\begin{array}{l}66(45.83) \\
78(54.17) \\
\end{array}$ & $\begin{array}{c}1.00 \text { (ref) } \\
1.33(0.75-2.37) \\
\end{array}$ & 0.33 \\
\hline & Recessive & $\begin{array}{c}\mathrm{C} / \mathrm{C}-\mathrm{C} / \mathrm{T} \\
\mathrm{T} / \mathrm{T} \\
\end{array}$ & $\begin{array}{l}83(83) \\
17(17) \\
\end{array}$ & $\begin{array}{c}131(90.96) \\
13(9.04) \\
\end{array}$ & $\begin{array}{c}1.00 \text { (ref) } \\
2.53(1.08-5.93) \\
\end{array}$ & $0.03^{*}$ \\
\hline & Overdominant & $\begin{array}{c}\mathrm{C} / \mathrm{C}-\mathrm{T} / \mathrm{T} \\
\mathrm{C} / \mathrm{T} \\
\end{array}$ & $\begin{array}{l}52(52) \\
48(48) \\
\end{array}$ & $\begin{array}{l}79(54.9) \\
65(45.1) \\
\end{array}$ & $\begin{array}{c}1.0(\mathrm{ref}) \\
0.86(0.49-1.53) \\
\end{array}$ & 0.61 \\
\hline & Additive & - & - & - & $1.46(0.96-2.23)$ & 0.07 \\
\hline \multirow[t]{5}{*}{ MTR A2756G } & Codominant & $\begin{array}{c}\text { AA } \\
\text { AG } \\
\text { GG } \\
\text { Allele A } \\
\text { Allele G } \\
\text { HWE test } \\
\end{array}$ & $\begin{array}{c}68(68) \\
31(31) \\
1(1) \\
167(83.5) \\
33(16.5) \\
P=0.21 \\
\end{array}$ & $\begin{array}{c}88(61.11) \\
50(34.72) \\
6(4.17) \\
226(78.4) \\
62(21.6) \\
P=0.73 \\
\end{array}$ & $\begin{array}{c}1.00 \text { (ref) } \\
1.01(0.55-1.85) \\
0.24(0.03-2.17)\end{array}$ & 0.35 \\
\hline & Dominant & $\begin{array}{c}\mathrm{A} / \mathrm{A} \\
\mathrm{A} / \mathrm{G}-\mathrm{G} / \mathrm{G}\end{array}$ & $\begin{array}{l}68(68) \\
32(32)\end{array}$ & $\begin{array}{l}88(61.11) \\
56(38.89)\end{array}$ & $\begin{array}{c}1.00 \text { (ref) } \\
0.91(0.51-1.65)\end{array}$ & 0.77 \\
\hline & Recessive & $\begin{array}{c}\mathrm{A} / \mathrm{A}-\mathrm{A} / \mathrm{G} \\
\mathrm{G} / \mathrm{G}\end{array}$ & $\begin{array}{l}99(99) \\
01(01) \\
\end{array}$ & $\begin{array}{c}138(95.83) \\
6(4.17) \\
\end{array}$ & $\begin{array}{c}1.0 \text { (ref) } \\
0.24(0.03-2.15) \\
\end{array}$ & 0.15 \\
\hline & Overdominant & $\begin{array}{c}\mathrm{A} / \mathrm{A}-\mathrm{G} / \mathrm{G} \\
\mathrm{A} / \mathrm{G}\end{array}$ & $\begin{array}{l}69(69) \\
31(31) \\
\end{array}$ & $\begin{array}{l}94(65.3) \\
50(34.7) \\
\end{array}$ & $\begin{array}{c}1.0(\mathrm{ref}) \\
1.06(0.58-1.94)\end{array}$ & 0.84 \\
\hline & Additive & - & - & - & $0.83(0.50-1.40)$ & 0.49 \\
\hline
\end{tabular}

$\mathrm{OR}=$ odds ratio; adjusted for age, alcohol and smoking consumption, BMI (Body-mass index) and polymorphisms; HWE $=$ Hardy-Weinberg equilibrium; ${ }^{*}$ P values statistically significant.

Hardy-Weinberg equilibrium for thyroid cancer and controls individuals showed that genotype frequencies were in equilibrium within the case $\left(\chi^{2}=2.02, \mathrm{P}=0.15\right)$ and control groups $\left(\chi^{2}=0.28, \mathrm{P}=0.59\right)$ for MTHFR C677T polymorphism. For MTR A2756G polymorphism, equilibrium was only observed in the control group $\left(\chi^{2}=0.11, \mathrm{P}=0.73\right)$; the thyroid cancer group presented disequilibrium $\left(\chi^{2}=4.38, \mathrm{P}=0.03\right)$ (Table 1). In Breast cancer patients and controls individuals, both polymorphisms were in equilibrium (MTHFR C677T case group: $\chi^{2}=0.006, \mathrm{P}=0.93$ and control group: $\chi^{2}=0.28, \mathrm{P}=0.59$; MTR A2756G case group: $\chi^{2}=1.56, \mathrm{P}=0.21$ and control group: $\chi^{2}=0.11, \mathrm{P}=0.73$ ) (Table 2).

Multiple logistic regression showed that tobacco consumption $(\mathrm{OR}=1.82 ; 95 \% \mathrm{CI}$ $=1.02-3.25 ; \mathrm{P}=0.04)$ and $\mathrm{BMI}(\mathrm{OR}=1.81 ; 95 \% \mathrm{CI}=1.00-3.25 ; \mathrm{P}=0.04)$ were risk factors for thyroid cancer development. On the other hand, patients 49 years and older, as well as alcohol drinking, were found to be unrelated to development of thyroid cancer. Individuals over 50 years of age $(\mathrm{OR}=3.14 ; 95 \% \mathrm{CI}=1.79-5.51 ; \mathrm{P}<0.001)$ and those that consume alcohol $(\mathrm{OR}=1.87 ; 95 \% \mathrm{CI}=1.05-3.34 ; \mathrm{P}=0.03)$ were more frequently found in breast cancer as compared to the control group. There was no association between tobacco use and BMI and breast cancer development (Table 3).

Tables 4 and 5 show interaction analysis between MTHFR C677T and MTR A2756G polymorphisms and the variables studied (alcohol consumption, tobacco consumption, and BMI) with regard to risks for thyroid and breast cancers. There was no interaction between the variables in both types of cancers ( $\mathrm{P}$ value $\geq 0.05$ ). 
Table 3. Risk factors and odds ratio (OR) for thyroid and breast cancer.

\begin{tabular}{|c|c|c|c|c|c|}
\hline Cancer & Variable & Patients $(\mathrm{N}=100)[\mathrm{N}(\%)]$ & Controls $(\mathrm{N}=144)[\mathrm{N}(\%)]$ & $\mathrm{OR}(95 \% \mathrm{CI})$ & $\mathrm{P}$ value \\
\hline \multirow[t]{12}{*}{ Thyroid cancer } & \multicolumn{3}{|l|}{ Age (years) } & \multirow{3}{*}{$\begin{array}{c}1.00(\mathrm{ref}) \\
1.43(0.84-2.45)\end{array}$} & \multirow[t]{3}{*}{0.19} \\
\hline & $<49$ & $44(44)$ & 77 (53.48) & & \\
\hline & $\geq 49$ & $56(56)$ & $67(46.52)$ & & \\
\hline & \multicolumn{3}{|l|}{ Alcohol consumption } & \multirow{3}{*}{$\begin{array}{c}1.00 \text { (ref) } \\
0.53(0.28-1.02)\end{array}$} & \multirow[t]{3}{*}{0.06} \\
\hline & No & $81(81)$ & $103(71.58)$ & & \\
\hline & Yes & $19(19)$ & $41(28.42)$ & & \\
\hline & \multicolumn{3}{|l|}{ Tobacco consumption } & \multirow{3}{*}{$\begin{array}{c}1.00 \text { (ref) } \\
1.82(1.02-3.25)\end{array}$} & \multirow[t]{3}{*}{$0.04^{*}$} \\
\hline & No & $62(62)$ & $106(73.62)$ & & \\
\hline & Yes & $38(38)$ & $38(26.38)$ & & \\
\hline & BMI & & & \multirow{3}{*}{$\begin{array}{c}1.00 \text { (ref) } \\
1.81(1.00-3.25)\end{array}$} & \multirow[t]{3}{*}{$0.04^{*}$} \\
\hline & $<25.0$ & $26(26)$ & $54(37.5)$ & & \\
\hline & $\geq 25.0$ & $74(74)$ & $90(62.5)$ & & \\
\hline \multirow[t]{12}{*}{ Breast cancer } & Age (years) & & & \multirow{3}{*}{$\begin{array}{c}1.00 \text { (ref) } \\
3.14(1.79-5.51)\end{array}$} & \multirow[t]{3}{*}{$<0.001 *$} \\
\hline & $<50$ & $32(32)$ & $84(58.34)$ & & \\
\hline & $\geq 50$ & $68(68)$ & $60(41.66)$ & & \\
\hline & \multicolumn{3}{|l|}{ Alcohol consumption } & \multirow{3}{*}{$\begin{array}{c}1.00 \text { (ref) } \\
1.87(1.05-3.34)\end{array}$} & \multirow[t]{3}{*}{$0.03^{*}$} \\
\hline & No & $54(54)$ & $103(71.58)$ & & \\
\hline & Yes & $46(46)$ & $41(28.42)$ & & \\
\hline & \multicolumn{3}{|l|}{ Tobacco consumption } & \multirow{3}{*}{$\begin{array}{c}1.00 \text { (ref) } \\
1.28(0.70-2.35)\end{array}$} & \multirow[t]{3}{*}{0.42} \\
\hline & No & $64(64)$ & $106(73.62)$ & & \\
\hline & Yes & $36(36)$ & $38(26.38)$ & & \\
\hline & \multicolumn{3}{|l|}{ BMI } & \multirow{3}{*}{$\begin{array}{c}1.00 \text { (ref) } \\
1.31(0.73-2.33)\end{array}$} & \multirow[t]{3}{*}{0.36} \\
\hline & $<25.0$ & $31(31)$ & $54(37.5)$ & & \\
\hline & $\geq 25.0$ & $69(69)$ & $90(62.5)$ & & \\
\hline
\end{tabular}

$\mathrm{OR}=$ odds ratio; adjusted for age, alcohol and smoking consumption, BMI (Body-mass index) and polymorphisms in the recessive model; *P value statistically significant.

Table 4. Interaction between MTHFR C677T and MTR A2756G polymorphisms and alcohol and tobacco consumption and BMI on the risk of thyroid cancer.

\begin{tabular}{|c|c|c|c|c|}
\hline & \multicolumn{2}{|c|}{ MTHFR C677T } & \multicolumn{2}{|c|}{$M T R$ A2756G } \\
\hline & $\mathrm{CC} / \mathrm{CT}$ & TT & $\mathrm{AA} / \mathrm{AG}$ & GG \\
\hline Alcohol consumption & $\mathrm{N}(\%)$ & $\mathrm{N}(\%)$ & $\mathrm{N}(\%)$ & $\mathrm{N}(\%)$ \\
\hline \multicolumn{5}{|l|}{ No } \\
\hline Case & $65(65)$ & $15(15)$ & $73(73)$ & $07(07)$ \\
\hline Control & $93(64.6)$ & $10(6.9)$ & $98(68)$ & $05(3.5)$ \\
\hline OR $(95 \% \mathrm{CI})$ & 1.00 & $2.50(1.03-6.04)$ & 1.00 & $1.64(0.49-5.51)$ \\
\hline \multicolumn{5}{|l|}{ Yes } \\
\hline Case & $16(16)$ & $04(04)$ & $18(18)$ & $02(02)$ \\
\hline Control & $38(26.4)$ & $03(2.1)$ & $40(27.8)$ & $01(0.7)$ \\
\hline $\mathrm{OR}(95 \% \mathrm{CI})$ & $0.56(0.28-1.12)$ & $1.71(0.36-8.17)$ & $0.56(0.29-1.07)$ & $2.32(0.20-26.98)$ \\
\hline P interaction & \multicolumn{2}{|c|}{0.84} & \multicolumn{2}{|c|}{0.50} \\
\hline \multicolumn{5}{|l|}{ Tobacco consumption } \\
\hline \multicolumn{5}{|l|}{ No } \\
\hline Case & $47(47)$ & $15(15)$ & $56(56)$ & $06(06)$ \\
\hline Control & $95(65.9)$ & $11(7.6)$ & $104(72.2)$ & $02(1.4)$ \\
\hline OR $(95 \% \mathrm{CI})$ & 1.00 & $2.64(1.11-6.26)$ & 1.00 & $5.52(1.06-28.73)$ \\
\hline \multicolumn{5}{|l|}{ Yes } \\
\hline Case & $34(34)$ & $04(04)$ & $35(35)$ & $03(03)$ \\
\hline Control & $36(25)$ & $02(1.4)$ & 34 (23.6) & $04(2.8)$ \\
\hline $\mathrm{OR}(95 \% \mathrm{CI})$ & $1.94(1.06-3.53)$ & $4.83(0.83-28.20)$ & $2.02(1.11-3.65)$ & $1.30(0.28-6.08)$ \\
\hline $\mathrm{P}$ interaction & \multicolumn{2}{|c|}{0.96} & \multicolumn{2}{|c|}{0.06} \\
\hline \multicolumn{5}{|l|}{ Body-mass index } \\
\hline \multicolumn{5}{|l|}{$<25 \mathrm{~kg} / \mathrm{m}^{2}$} \\
\hline Case & $21(21)$ & $05(05)$ & $24(24)$ & $02(02)$ \\
\hline Control & $49(34)$ & $05(3.5)$ & $52(36.1)$ & $02(1.4)$ \\
\hline OR $(95 \% \mathrm{CI})$ & 1.00 & $2.48(0.64-9.66)$ & 1.00 & $1.60(0.21-12.45)$ \\
\hline \multicolumn{5}{|l|}{$\geq 25 \mathrm{~kg} / \mathrm{m}^{2}$} \\
\hline Case & $60(60)$ & $14(14)$ & $67(67)$ & $07(07)$ \\
\hline Control & $82(56.9)$ & $08(5.6)$ & $86(59.7)$ & $04(2.8)$ \\
\hline $\mathrm{OR}(95 \% \mathrm{CI})$ & $1.67(0.90-3.11)$ & $4.46(1.60-12.42)$ & $1.65(0.92-2.97)$ & $3.56(0.93-13.71)$ \\
\hline P interaction & & & & \\
\hline
\end{tabular}

$\mathrm{OR}=$ odds ratio; adjusted for age, alcohol consumption tobacco consumption and Body-mass index. *P value statistically significant. 
Table 5. Interaction between MTHFR C677T and MTR A2756G polymorphisms and alcohol and tobacco consumption and BMI on the risk of breast cancer.

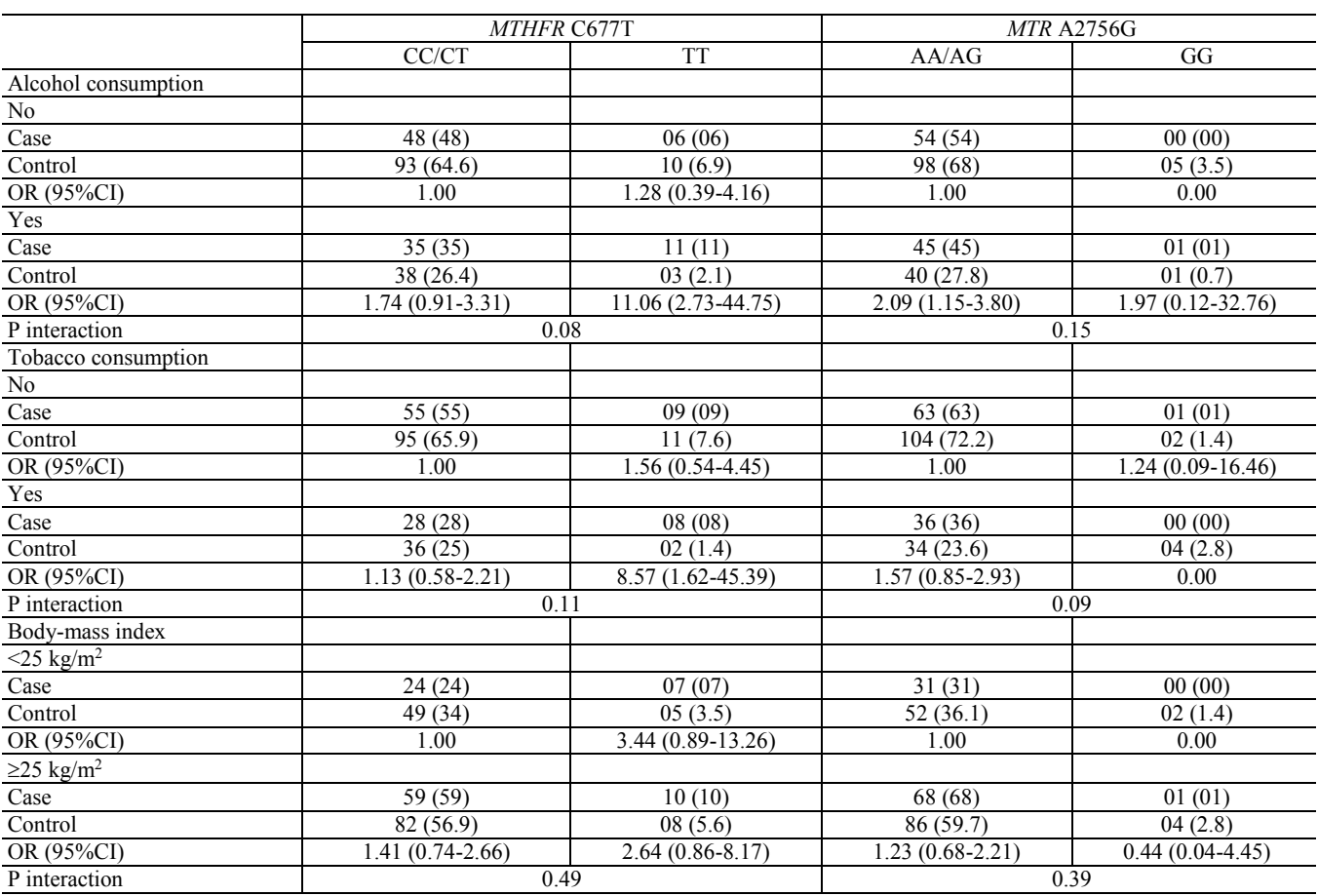

$\mathrm{OR}=$ odds ratio; adjusted for age, alcohol consumption tobacco consumption and Body-mass index. *P value statistically significant.

\section{DISCUSSION}

In the present study, we evaluated the association between MTHFR C677T and MTR A2756G polymorphism and thyroid and breast cancers. We also investigated the interactions between polymorphisms and possible risk factors for the referred disorders. We found an association between the MTHFR C677T polymorphism variant genotype (TT) and increased risk to both cancers. In addition, tobacco consumption and BMI were also associated with thyroid cancer development. Old age ( $\geq 50$ years) and alcohol consumption were observed to be positively associated with breast cancer development.

Furthermore, in our study, we have not observed the Hardy Weinberg equilibrium in the thyroid cancer group. This is due to random selection samples, models used, and complexity of disease that involved both biological and genetic features (Wittke-Thompson et al., 2005). Some polymorphism in the folate pathway alters enzyme activities. It interfere in DNA methylation, in the synthesis of purines and pyrimidine, as well as in the genomic instability, induces higher susceptibility to cancer development (Carvalho Barbosa et al., 2012; Weiner et al., 2012). The MTHFR gene reduces enzymatic activity by limiting the conversion of 5,10 methylenetetrahydrofolate to 5-MTHFR, which is the only form of folate required for DNA methylation reaction. This reduction is important as it leads to cancer susceptibility. 
DNA hypomethylation is associated with several types of cancers, and occurs as a result of a decrease in the concentration of 5-MTHFR (Yin et al., 2004; Alshatwi, 2010; Akilzhanova et al., 2013). The association of the recessive model (genotype 677TT) MTHFR gene with increased risk for thyroid and breast cancers was observed in the present study $(\mathrm{OR}=2.50$; $95 \% \mathrm{CI}=1.15-5.46 ; \mathrm{P}=0.02)$ and $(\mathrm{OR}=2.53 ; 95 \% \mathrm{CI}=1.08-5.93 ; \mathrm{P}=0.03)$, respectively. A study by Ozdemir et al., 2012 involving 60 cases and 50 controls found an increased risk for thyroid cancer by 2.33 -fold in individuals with the homozygous recessive genotype (677TT). A similar risk (2.08-fold) for the same genotype was also reported by Fard-Esfahani et al. (2011) in a study involving 154 cases and 198 controls. Both studies included men and women. In a breast cancer case-control study conducted in Chinese women, an increased risk for cancer development was found in those carrying the 677TT genotype (He et al., 2014; Jiang-hua et al., 2014; Weiwei et al., 2014). These results were all in agreement with our present findings.

The genotype $677 \mathrm{CT}+\mathrm{TT}$ and $677 \mathrm{CT}$ showed an increased risk for breast cancer by 1.2-fold and 1.3-fold in Kazakhstan's population, respectively (Akilzhanova et al., 2013). Another study in a Moroccan population conducted by Diakite et al. (2012) involving 96 women found an association between at least one polymorphic allele and breast cancer increased risk, contrary to our findings. In our study, we found no statistically significant differences between $677 \mathrm{CT}+\mathrm{TT}$ and 677CT genotypes in both types of cancers studied. Our results were similar to other studies addressing thyroid and breast cancers (Kotsopoulos et al., 2008; Kweon et al., 2014). For the MTR A2756G polymorphism, ours results have shown no association between this SNP and thyroid and breast cancers, which agreed with the results from 4 case-control breast cancer studies (Naushad et al., 2011; He et al., 2014; Jiang-hua et al., 2014; Weiwei et al., 2014). Meta-analyses by Zhong et al. (2013) and Weiner et al. (2012) also found no association between MTR A2756G polymorphism and breast cancer development. However, a Brazilian case-control study performed in the Northeast region and a study that evaluated the Iranian population found an association of at least one polymorphic allele (2756G) in breast cancer (Carvalho Barbosa et al., 2012; Hosseini, 2013). This polymorphism is associated with a reduction in the MTR enzyme, leading to elevated homocysteine level and DNA hypomethylation (Hosseini, 2013). Studies on polymorphisms and cancer risk presented controversial results due to several factors such as a measurement sample, ethnicity and population studied, hormones, and environmental factors such as folate intake (Diakite et al., 2012; Gong et al., 2015).

Many studies have shown the importance of smoking habit in cancer development (Galbiatti et al., 2010; Rodrigues et al., 2010). The association between tobacco consumption and thyroid cancer was also found in this study $(\mathrm{OR}=1.82 ; 95 \% \mathrm{CI}=1.02-3.25, \mathrm{P}=0.04)$. This was in agreement with a meta-analysis consisting of 25 case-controls studies and six cohort studies, which showed that tobacco consumption is a predictive factor to several thyroid malignancies (Cho and Kim, 2014). Another factor described in literature as a predictor for development of several types of cancers is obesity. This was found to be associated with thyroid cancer in our study $(\mathrm{OR}=1.81 ; 95 \% \mathrm{CI}=1.00-3.25, \mathrm{P}=0.04)$. Some studies have also confirmed an association between obesity and increased risk for thyroid cancer. Obesity may influence tumor size and extrathyroidal invasion, and may increase aggressiveness and metastasis of cancer (Kim et al., 2015; Ma et al., 2015). In agreement with our study, Guignard et al. (2007) found no evidence between alcohol consumption and thyroid cancer risk in New Caledonia (Oceania) population.

In this study, we found that women aged 50 years and above were at risk for breast 
cancer development. Increased age has been strongly related to postmenopausal period in accordance with literatures (Sangrajrang et al., 2010; He et al., 2014). Alcohol consumption was found to be a predictor factor for breast cancer development in women $(\mathrm{OR}=1.87$; $95 \% \mathrm{CI}=1.05-3.34 ; \mathrm{P}=0.03)$. This association was also confirmed in two case-control studies carried out in China $(\mathrm{P}=0.002)$ (Jiang-hua et al., 2014) and Malmo (South of Sweden) ( $\mathrm{P}$ $=0.001)$ (Ericson et al., 2009). The intake of alcoholic beverages causes poor absorption of B-complex vitamins, modifies folate metabolism, induces oxidative injury, and damages DNA (Sellers et al., 2001). Similar to our results, tobacco consumption and BMI was found to be unassociated with breast cancer in other studies as well (Ericson et al., 2009; Sangrajrang et al., 2010; Gong et al., 2015). One limiting factor in our study was the relatively small sample size, as the implementation period was relatively short. Nevertheless, results from our study, in combination with others studies, should provide a comprehensive understanding between the folate pathway and both breast and thyroid cancer. It is noteworthy to emphasize the fact there is very little literature reporting on the association between folate pathway and thyroid cancer.

Our case-control study showed that women presented with the MTHFR 677TT genotype has an increased risk for thyroid and breast cancers. Additionally, tobacco consumption and obesity are associated with thyroid cancer development. Alcohol consumption was found to be associated with breast cancer development in women greater than 50 years of age. Further investigations on gene-gene interactions between folate metabolism and cancer development need to be carried out in other populations to gain greater understandings of the effect of genetic polymorphisms on risk of breast and thyroid cancers.

\section{Conflicts of interest}

The authors declare no conflict of interest.

\section{ACKNOWLEDGMENTS}

We appreciate the CAPES, CNPq (Process CNPq universal \#470833/2012-2), and FAPESP (\#2010/12930-4 and \#2012/14781-1) for the financial support. We also thank, the Medical School of São José do Rio Preto, FAMERP and Medical School Foundation, and FUNFARME for institutional support. Lastly, we want to thank the Otorhinolaryngology and Head and Neck Surgery Department and Gyneacologic and Obstetric Services of Hospital de Base, São José do Rio Preto.

\section{REFERENCES}

Ahrendt SA, Chow JT, Yang SC, Wu L, et al. (2000). Alcohol consumption and cigarette smoking increase the frequency of p53 mutations in non-small cell lung cancer. Cancer Res. 60: 3155-3159.

Akilzhanova A, Nurkina Z, Momynaliev K, Ramanculov E, et al. (2013). Genetic profile and determinants of homocysteine levels in Kazakhstan patients with breast cancer. Anticancer Res. 33: 4049-4059.

Alshatwi AA (2010). Breast cancer risk, dietary intake, and methylenetetrahydrofolate reductase (MTHFR)single nucleotide polymorphisms. Food Chem. Toxicol. 48: 1881-1885. http://dx.doi.org/10.1016/j.fct.2010.04.028

Carpenter CL, Morgenstern H and London SJ (1998). Alcoholic beverage consumption and lung cancer risk among residents of Los Angeles County. J. Nutr. 128: 694-700.

Carvalho Barbosa RdeC, Menezes DC, Freire TF, Sales DC, et al. (2012). Associations of polymorphisms of folate cycle enzymes and risk of breast cancer in a Brazilian population are age dependent. Mol. Biol. Rep. 39: 4899-4907. http:// dx.doi.org/10.1007/s11033-011-1285-1 
Cho YA and Kim J (2014). Thyroid cancer risk and smoking status: a meta-analysis. Cancer Causes Control Pap. Symp. 25: 1187-1195.

Diakite B, Tazzite A, Hamzi K, Jouhadi H, et al. (2012). Methylenetetrahydrofolate reductase C677T polymorphism and breast cancer risk in Moroccan women. Afr. Health Sci. 12: 204-209.

Ericson UC, Ivarsson MI, Sonestedt E, Gullberg B, et al. (2009). Increased breast cancer risk at high plasma folate concentrations among women with the MTHFR 677T allele. Am. J. Clin. Nutr. 90: 1380-1389. http://dx.doi. org/10.3945/ajen.2009.28064

Fard-Esfahani P, Fard-Esfahani A, Saidi P, Fayaz S, et al. (2011). An increased risk of differentiated thyroid carcinoma in Iran with the 677C®T homozygous polymorphism in the MTHFR Gene. Cancer Epidemiol. 35: 56-58. http://dx.doi. org/10.1016/j.canep.2010.10.001

Galbiatti AL, Ruiz MT, Biselli-Chicote PM, Raposo LS, et al. (2010). 5-Methyltetrahydrofolate-homocysteine methyltransferase gene polymorphism (MTR) and risk of head and neck cancer. Braz. J. Med. Biol. Res. 43: 445450. http://dx.doi.org/10.1590/S0100-879X2010007500034

Gong Z, Yao S, Zirpoli G, David Cheng TY, et al. (2015). Genetic variants in one-carbon metabolism genes and breast cancer risk in European American and African American women. Int. J. Cancer 137: 666-677. http://dx.doi. org/10.1002/ijc. 29434

Guignard R, Truong T, Rougier Y, Baron-Dubourdieu D, et al. (2007). Alcohol drinking, tobacco smoking, and anthropometric characteristics as risk factors for thyroid cancer: a countrywide case-control study in New Caledonia. Am. J. Epidemiol. 166: 1140-1149. http://dx.doi.org/10.1093/aje/kwm204

He JM, Pu YD, Wu YJ, Qin R, et al. (2014). Association between dietary intake of folate and MTHFR and MTR genotype with risk of breast cancer. Genet. Mol. Res. 13: 8925-8931. http://dx.doi.org/10.4238/2014.October.31.7

Hosseini M (2013). Role of polymorphism of methyltetrahydrofolate-homocysteine methyltransferase (MTR) A2756G and breast cancer risk. Pol. J. Pathol. 64: 191-195. http://dx.doi.org/10.5114/pjp.2013.38138

INCA (Instituto Nacional do Câncer Estimativa) (2014). Incidência de câncer no Brasil. Available at [http://www. saude.sp.gov.br/resources/ses/perfil/gestor/homepage/outros-destaques/estimativa-de-incidencia-de-cancer-2014/ estimativa cancer 24042014.pdf]. Accessed July 14, 2015.

James FR, Wootton S, Jackson A, Wiseman M, et al. (2015). Obesity in breast cancer--what is the risk factor? Eur. J. Cancer 51: 705-720. http://dx.doi.org/10.1016/j.ejca.2015.01.057

Kim SH, Park HS, Kim KH, Yoo H, et al. (2015). Correlation between obesity and clinicopathological factors in patients with papillary thyroid cancer. Surg. Today 45: 723-729. http://dx.doi.org/10.1007/s00595-014-0984-3

Kjaerheim K, Gaard M and Andersen A (1998). The role of alcohol, tobacco, and dietary factors in upper aerogastric tract cancers: a prospective study of 10,900 Norwegian men. Cancer Causes Control Pap. Symp. 9: 99-108.

Kotsopoulos J, Zhang WW, Zhang S, McCready D, et al. (2008). Polymorphisms in folate metabolizing enzymes and transport proteins and the risk of breast cancer. Breast Cancer Res. Treat. 112: 585-593. http://dx.doi.org/10.1007/ $\underline{\text { s10549-008-9895-6 }}$

Kweon SS, Shin MH, Kim HN, Kim SH, et al. (2014). Polymorphisms of methylenetetrahydrofolate reductase and glutathione S-transferase are not associated with the risk of papillary thyroid cancer in Korean population. Mol. Biol. Rep. 41: 3793-3799. http://dx.doi.org/10.1007/s11033-014-3245-z

Ma J, Huang M, Wang L, Ye W, et al. (2015). Obesity and risk of thyroid cancer: evidence from a meta-analysis of 21 observational studies. Med. Sci. Monit. 21: 283-291. http://dx.doi.org/10.12659/MSM.892035

Miller SA, Dykes DD and Polesky HF (1988). A simple salting out procedure for extracting DNA from human nucleated cells. Nucleic Acids Res. 16: 1215. http://dx.doi.org/10.1093/nar/16.3.1215

Naushad SM, Pavani A, Digumarti RR, Gottumukkala SR, et al. (2011). Epistatic interactions between loci of one-carbon metabolism modulate susceptibility to breast cancer. Mol. Biol. Rep. 38: 4893-4901. http://dx.doi.org/10.1007/ s11033-010-0631-z

Ozdemir S, Silan F, Hasbek Z, Uludag A, et al. (2012). Increased T-allele frequency of $677 \mathrm{C}>\mathrm{T}$ polymorphism in the methylenetetrahydrofolate reductase gene in differentiated thyroid carcinoma. Genet. Test. Mol. Biomarkers 16: 780784. http://dx.doi.org/10.1089/gtmb.2011.0347

Jiang-hua Q, De-Chuang J, Zhen-Duo L, Shu-de C, et al. (2014). Association of methylenetetrahydrofolate reductase and methionine synthase polymorphisms with breast cancer risk and interaction with folate, vitamin B6, and vitamin B 12 intakes. Tumour Biol. 35: 11895-11901. http://dx.doi.org/10.1007/s13277-014-2456-1

Rodrigues JO, Galbiatti ALS, Ruiz MT, Raposo LS, et al. (2010). Polymorphism of methylenetetrahydrofolate reductase (MTHFR) gene and risk of head and neck squamous cell carcinoma. Rev. Bras. Otorrinolaringol. (Engl. Ed) 76: 776-782.

Sangrajrang S, Sato Y, Sakamoto H, Ohnami S, et al. (2010). Genetic polymorphisms in folate and alcohol metabolism 
and breast cancer risk: a case-control study in Thai women. Breast Cancer Res. Treat. 123: 885-893. http://dx.doi. org/10.1007/s10549-010-0804-4

Sellers TA, Kushi LH, Cerhan JR, Vierkant RA, et al. (2001). Dietary folate intake, alcohol, and risk of breast cancer in a prospective study of postmenopausal women. Epidemiology 12: 420-428. http://dx.doi.org/10.1097/00001648200107000-00012

Taflin H, Wettergren Y, Odin E, Carlsson G, et al. (2014). Folate levels and polymorphisms in the genes MTHFR, MTR, and TS in colorectal cancer. Clin. Med. Insights Oncol. 8: 15-20. http://dx.doi.org/10.4137/CMO.S12701

Weiner AS, Boyarskikh UA, Voronina EN, Selezneva IA, et al. (2012). Polymorphisms in the folate-metabolizing genes MTR, MTRR, and CBS and breast cancer risk. Cancer Epidemiol. 36: e95-e100. http://dx.doi.org/10.1016/j. canep.2011.11.010

Wittke-Thompson JK, Pluzhnikov A and Cox NJ (2005). Rational inferences about departures from Hardy-Weinberg equilibrium. Am. J. Hum. Genet. 76: 967-986. http://dx.doi.org/10.1086/430507

Yang D, Baumgartner RN, Slattery ML, Wang C, et al. (2013). Dietary intake of folate, B-vitamins and methionine and breast cancer risk among Hispanic and non-Hispanic white women. PLoS One 8: e54495. http://dx.doi.org/10.1371/ journal.pone.0054495

Yang YM, Zhang TT, Yuan L and Ren Y (2014). The association between the C677T polymorphism in MTHFR gene and the risk of thyroid cancer: a meta-analysis. Eur. Rev. Med. Pharmacol. Sci. 18: 2097-2101.

Yin G, Kono S, Toyomura K, Hagiwara T, et al. (2004). Methylenetetrahydrofolate reductase C677T and A1298C polymorphisms and colorectal cancer: the Fukuoka Colorectal Cancer Study. Cancer Sci. 95: 908-913. http://dx.doi. org/10.1111/j.1349-7006.2004.tb02201.x

Weiwei Z, Liping C and Dequan L (2014). Association between dietary intake of folate, vitamin B6, B12 \& MTHFR, MTR Genotype and breast cancer risk. Pak. J. Med. Sci. 30: 106-110.

Zhong S, Xu J, Li W, Chen Z, et al. (2013). Methionine synthase A2756G polymorphism and breast cancer risk: an up-todate meta-analysis. Gene 527: 510-515. http://dx.doi.org/10.1016/j.gene.2013.06.054 